\title{
QUANTIFICATION OF TOPOLOGICAL CONCEPTS USING IDEALS
}

\author{
ROBERT LOWEN and BART WINDELS
}

(Received 19 February 2000)

\begin{abstract}
We introduce certain ideals of real-valued functions as a natural generalization of filters. We show that these ideals establish a canonical framework for the quantification of topological concepts, such as closedness, adherence, and compactness, in the setting of approach spaces.
\end{abstract}

2000 Mathematics Subject Classification. 54A05, 54D30.

1. Introduction. In [3], Kuratowski introduced what he called the measure of noncompactness for complete metric spaces. The purely topological concept of compactness was quantified in the setting of metric spaces in order to measure the discrepancy a metric space may have from being compact. Since then several variants, such as Hausdorff's ball measure of noncompactness have been introduced. For an extensive account on applications of these measures in the setting of Banach spaces, we refer to Banaś and Goebel [1]. All what these measures have in common is that they involve a (pseudo-)metric space.

The introduction of approach spaces (see [5]), established a more general setting for the quantification of topological concepts. Approach spaces, which are a unification of topological spaces and metric spaces, express both qualitative and quantitative information. If these kinds of information are combined in a relevant (though canonical) way, then the numerical information can be used to express to what extent some qualitative aspect is or is not fulfilled. In [4], it is shown that compactness of topological spaces and total boundedness of metric spaces are special instances of a unifying concept for approach spaces, yielding a measure of compactness for approach spaces. Moreover, this measure is a generalization of the Hausdorff measure for metric spaces, mentioned above. Also, a measure of connectedness is defined, generalizing connectedness and Cantor-connectedness. For a recent and full account on approach spaces, we refer to [6].

Consider for instance, the following example in [6]. Let $X$ be a separable metrizable space and suppose the set $\mathcal{M}(X)$ of probability measures on $X$ is equipped with the so-called weak approach structure (which is a canonical generalization of the weak topology on $\mathcal{M}(X))$. Further, let $\mathscr{H}$ be a weakly compact subset of $\mathcal{M}(X)$ and let $\mathscr{K}$ be an arbitrary subset of $\mathcal{M}(X)$. Then the collection of contaminated probability measures

$$
\mathscr{H}^{\prime}:=\{(1-\alpha) P+\alpha Q \mid P \in \mathscr{H}, Q \in \mathscr{K}, \alpha \leq \varepsilon\}
$$

need no longer be compact, although from a statistical point of view, $\mathscr{H}$ and $\mathscr{H}^{\prime}$ are 
indiscernible for $\varepsilon$ sufficiently small. The fact that $\mathscr{H}^{\prime}$ is almost compact can be expressed formally by saying that the measure of compactness is $\mu_{c}\left(\mathscr{H}^{\prime}\right) \leq 2 \varepsilon$ (see [6, Example 6.1.16]), that is, $\mathscr{H}^{\prime}$ is weakly compact "up to $2 \varepsilon . "$

Using the measure of compactness we regain a lot of information compared to the classical situation of topological spaces. Nevertheless, we can do better. It is intuitively quite clear that, in general, the probability measure $(1-\varepsilon) P+\varepsilon Q$ contributes more to the noncompactness of $\mathscr{H}^{\prime}$ than $(1-(\varepsilon / 2)) P+\varepsilon / 2 Q$ does. In fact, every element in $\mathcal{H}^{\prime}$ causes $\mu_{c}\left(\mathscr{H}^{\prime}\right)$ to deviate a certain amount from zero. So we can consider functions from $\mathscr{H}^{\prime}$ to $[0, \infty]$, mapping every element in $\mathscr{H}^{\prime}$ to a number that equals (or is smaller than) this deviation. In the sequel, we will call such a function compact.

To that end, we need a numerification of filters, called approach ideals, which will be the subject of the first section, where we will also introduce prime approach ideals (generalizing ultrafilters).

2. Approach ideals. In [5, 8], it is shown that if one wants to formulate canonical numerifications of topological or uniform concepts, it is useful to consider functions in $[0, \infty]^{X}$ instead of subsets of $X$, pointwise order instead of inclusion, ideals of functions instead of filters of sets and so forth. Consequently, we will introduce the following quantification of filters.

Definition 2.1. Let $X$ be a set. An approach ideal (a-ideal, for short) on $X$ is an ideal $\mathfrak{f}$ of functions in $[0, \infty]^{X}$ such that $\infty \notin \mathfrak{f}$.

If a collection $\mathfrak{G}$ of functions in $[0, \infty]^{X}$ not containing $\infty$, satisfies only the condition that for all $\phi_{1}, \phi_{2} \in \mathfrak{G}$, there exists $\phi_{3} \in \mathfrak{G}: \phi_{1} \vee \phi_{2} \leq \phi_{3}$, that is, if $\mathfrak{G}$ is an ideal basis, then $\mathbb{G}$ is called an approach ideal basis. The approach ideal generated by $\mathscr{G}$ is denoted by $\langle\mathfrak{6}\rangle$.

EXAMPLE 2.2. Let $\psi \in[0, \infty]^{X}$. Then

$$
\dot{\psi}:=\langle\{\psi\}\rangle=\{\phi \mid \phi \leq \psi\}
$$

is an a-ideal on $X$.

EXAMPLE 2.3. Let $\mathfrak{f}$ and $\mathfrak{G}$ be a-ideals on $X$ such that

$$
\forall \phi \in \mathfrak{f}, \forall \psi \in \mathfrak{G} \phi \vee \psi \neq \infty .
$$

Then

$$
\mathfrak{f} \vee \mathfrak{G}:=\{\phi \vee \psi \mid \phi \in \mathfrak{f}, \psi \in \mathfrak{G}\}, \quad \mathfrak{f}+\mathfrak{G}:=\{\phi+\psi \mid \phi \in \mathfrak{f}, \psi \in \mathfrak{G}\},
$$

are a-ideals on $X$.

EXAMPLE 2.4. Let $f: X \rightarrow Y$ be a function. If $f$ is an a-ideal on $X$, then

$$
f(\mathfrak{f}):=\left\langle\left\{\phi_{f} \mid \forall y \in Y: \phi_{f}(y)=\inf _{x \in f^{-1}\{y\}} \phi(x), \phi \in \mathfrak{f}\right\}\right\rangle
$$

is an a-ideal on $Y$. If $f$ is onto and $\mathscr{G}$ is an a-ideal on $Y$, then

$$
f^{-1}(\mathfrak{G}):=\langle\{\phi \circ f \mid \phi \in \mathfrak{G}\}\rangle
$$

is an a-ideal on $X$. 
In the setting of real-valued functions, the concept of ideals is in some situations too weak. Different ideals can be "almost equal" in the sense that the members of one ideal can be "uniformly approximated" by the members of another ideal and vice versa. Therefore, we will look at ideals which are maximal in this respect.

DEFinItion 2.5. Let $\mathfrak{f}$ be an a-ideal on $X$. Then $\mathfrak{f}$ is called saturated if and only if

$$
\left(\forall \varepsilon>0, \forall N<\infty, \exists \phi_{\varepsilon}^{N} \in \mathfrak{f}: \phi \wedge N \leq \phi_{\varepsilon}^{N}+\varepsilon\right) \Longrightarrow \phi \in \mathcal{f} .
$$

LEMMA 2.6. If $f$ is an a-ideal on $X$, then

$$
\hat{\mathfrak{f}}:=\left\{\phi \in[0, \infty]^{X} \mid \forall \varepsilon>0, \forall N<\infty, \exists \phi_{\varepsilon}^{N} \in \mathfrak{f}: \phi \wedge N \leq \phi_{\varepsilon}^{N}+\varepsilon\right\}
$$

is an a-ideal on $X$.

The a-ideal $\hat{\mathfrak{f}}$ is called the saturation of $\mathfrak{f}$. Clearly, $\mathfrak{f}$ is saturated if and only if $\mathfrak{f}=\hat{\mathfrak{f}}$.

Different useful topological and the like structures can be defined in the setting of a-ideals (cf. [7]). We give one important example, which we will pursue in the sequel.

DEFINITION 2.7 (see [5]). An approach system on $X$ is a family of saturated a-ideals $(\mathscr{A}(x))_{x \in X}$ such that for every $x \in X$

(A1) for all $\phi \in \mathscr{A}(x): \phi(x)=0$,

(A2) for all $\phi \in \mathscr{A}(x)$, for all $\varepsilon>0$, for all $N<\infty$, for all $z \in X$, there exists $\phi_{z} \in$ $\mathscr{A}(z)$ :

$$
\forall y, z \in X: \phi(z) \wedge N \leq \phi_{x}(y)+\phi_{y}(z)+\varepsilon .
$$

Then the pair $\left(X,(\mathscr{A}(x))_{x \in X}\right)$ is called an approach space.

In the sequel we will want to build filters with sections of functions $\phi \in[0, \infty]^{X}$, that is, sets of the form $\{\phi<\varepsilon\}:=\{x \in X \mid \phi(x)<\varepsilon\}$ for some $\varepsilon \in \mathbb{R}^{+}$. If $\mathcal{f}$ is an aideal, then we will call the smallest number $\varepsilon$ such that all sections $\{\phi<\varepsilon\}$ for $\phi \in f$ are nonempty, the height of $\mathfrak{f}$.

DeFinItion 2.8. Let $\mathfrak{f}$ be an a-ideal on $X$. Then

$$
h(\mathfrak{f}):=\sup _{\phi \in \mathfrak{f}} \inf _{x \in X} \phi(x)
$$

is called the height of $\mathfrak{f}$.

In the sequel, we will often abbreviate $\inf _{x \in X} \phi(x)$ by $\inf \phi$. If for each $\phi \in \mathfrak{f}$, $\inf \phi=0$, that is, if $h(\mathfrak{f})=0$, then $\mathfrak{f}$ is said to be of zero height. For instance, the a-ideals $\mathscr{A}(x)$ in an approach system are of zero height. If $h(\mathfrak{f})<\infty$, then $\mathfrak{f}$ is said to be of bounded height.

With an a-ideal on $X$ we can associate a sheaf of (ordinary) filters on $X$ in more than one canonical way.

Proposition 2.9. Let $\mathfrak{f}$ be an a-ideal on $X$ of bounded height $h$. Then for every $\varepsilon$ such that $h<\varepsilon \leq \infty$

$$
\mathfrak{f}_{\varepsilon}:=\langle\{\{\phi<\varepsilon\} \mid \phi \in \mathfrak{f}\}\rangle
$$


and for every $\varepsilon$ such that $h \leq \varepsilon<\infty$

$$
\mathfrak{f}^{\varepsilon}:=\langle\{\{\phi<\alpha\} \mid \phi \in \mathfrak{f}, \alpha>\varepsilon\}\rangle
$$

are filters on $X$.

Conversely, with a classical filter on $X$ we can associate in a natural way a-ideals of different heights.

Proposition 2.10. Let $\mathscr{F}_{F}$ be a filter on $X$ and let $\varepsilon<\infty$. Then

$$
\begin{aligned}
& \mathscr{F}_{\varepsilon}:=\left\{\phi \in[0, \infty]^{X} \mid\{\phi \leq \varepsilon\} \in \mathscr{F}\right\}, \\
& \mathscr{F}^{\varepsilon}:=\left\{\phi \in[0, \infty]^{X} \mid \forall \alpha>\varepsilon:\{\phi<\alpha\} \in \mathscr{F}_{\mathcal{F}}\right\}
\end{aligned}
$$

are a-ideals on $X$.

For every $A \subset X$ we define

$$
\theta_{A}: X \longrightarrow[0, \infty]: \begin{cases}x \longmapsto 0 & \text { if } x \in A, \\ x \longmapsto \infty & \text { if } x \notin A .\end{cases}
$$

Proposition 2.11. If $\mathscr{F}$ is a filter on $X$ and $\varepsilon<\infty$, then

$$
\mathscr{F}_{\varepsilon}=\left\langle\left\{\varepsilon+\theta_{F} \mid F \in \mathscr{F}_{F}\right\}\right\rangle .
$$

Proof. On the one hand, $\left\{\varepsilon+\theta_{F} \leq \varepsilon\right\}=F \in \mathscr{F}$. On the other hand, if $\{\phi \leq \varepsilon\} \in \mathscr{F}$, then $\phi \leq \varepsilon+\theta_{\{\phi \leq \varepsilon\}}$.

It is also possible to write down an explicit basis for $\mathscr{F}^{\varepsilon}$, as we did for $\mathscr{F}_{\varepsilon}$ in Proposition 2.11. The result is however quite involved, and can be inferred from [8].

EXAMPLE 2.12. Let $\left(X,(\mathscr{A}(x))_{x \in X}\right)$ be an approach space. Then the collection $\left(\mathscr{A}(x)^{\varepsilon}\right)_{x \in X, \varepsilon \in \mathbb{R}^{+}}$(defined as in Proposition 2.9) is a collection of filters on $X$ satisfying the following conditions for every $x \in X$ :

(B1) for all $\varepsilon \in \mathbb{R}^{+}$, for all $V \in \mathscr{A}(x)^{\varepsilon}: x \in V$,

(B2) for all $\varepsilon, \varepsilon^{\prime} \in \mathbb{R}^{+}$, for all $V \in \mathscr{A}(x)^{\varepsilon+\varepsilon^{\prime}}$, there exists $V_{x}^{\varepsilon} \in \mathscr{A}(x)^{\varepsilon}$, there exists $\left(V_{z}^{\varepsilon^{\prime}}\right)_{z} \in \prod_{z \in V_{x}^{\varepsilon}} \mathscr{A}(z)^{\varepsilon^{\prime}}: \cup_{z \in V_{x}^{\varepsilon}} V_{z}^{\varepsilon^{\prime}} \subset V$,

(B3) for all $\varepsilon \in \mathbb{R}^{+}: \mathscr{A}(x)^{\varepsilon}=\cup_{\varepsilon<\alpha} \mathscr{A}(x)^{\alpha}$, which can be shown using (A1), (A2) (see Definition 2.7 and Proposition 2.14(d)).

Conversely, if $\left(\mathscr{F}_{x}^{\varepsilon}\right)_{x \in X, \varepsilon \in \mathbb{R}^{+}}$is a collection of filters satisfying the above conditions, then

$$
\mathscr{A}(x):=\left\{\phi \mid \forall \varepsilon \in \mathbb{R}^{+}, \forall \alpha>\varepsilon:\{\phi<\alpha\} \in \mathscr{F}_{x}^{\varepsilon}\right\} \quad \text { (for every } x \in X \text { ) }
$$

defines an approach system such that for every $\varepsilon \in \mathbb{R}^{+}$we have $\mathscr{A}(x)^{\varepsilon}=\mathscr{F}_{x}^{\varepsilon}$. This means that an approach space can be described by a sheaf of pre-neighbourhood filters at every point $x \in X$ satisfying the quantified open kernel condition (B2).

In the sequel, we will denote the pre-closure operator associated with the pretopology $\left(\mathscr{A}(x)^{\varepsilon}\right)_{x \in X}$ by cl $\mathrm{cl}_{\varepsilon}$. Sometimes we will consider an approach space $\left(X,\left(\mathrm{cl}_{\varepsilon}\right)_{\varepsilon}\right)$ in terms of these pre-closure operators instead of the equivalent structure $(X$, $\left.(\mathscr{A}(x))_{x \in X}\right)$. 
EXAMPLE 2.13 (see [5]). Let $(\mathcal{N}(x))_{x \in X}$ be a family of neighbourhood filters on $X$, turning $X$ into a topological space. Then $\left(\mathcal{N}(x)_{0}\right)_{x \in X}$ is an approach structure on $X$. This construction yields an embedding functor from Top into Ap (the category of approach spaces and contractions). Moreover, this embedding is coreflective, the coreflection of any $(\mathscr{A}(x))_{x \in X}$ being $\left(\mathscr{A}(x)^{0}\right)_{x \in X}$.

Proposition 2.14. Let $\mathfrak{f}$ and $\mathfrak{G}$ be a-ideals on $X$, let $\mathscr{F}$ be a filter on $X$ and let $\varepsilon<\infty$. Then we have the following:

(a) If $\mathfrak{f} \subset \mathfrak{6}$, then $h(\mathfrak{f}) \leq h(\mathfrak{G})$.

(b) $h(\hat{\mathfrak{f}})=h(\mathfrak{f})$.

(c) $h\left(\mathscr{F}_{\varepsilon}\right)=\varepsilon$.

(d) $h\left(\mathscr{F}^{\varepsilon}\right)=\varepsilon$.

(e) If $\varepsilon<h(\mathfrak{f})$ and $A \in \mathfrak{f}_{\infty}$, then $\varepsilon+\theta_{A} \in \mathfrak{f}$.

Proof. Immediate.

Proposition 2.15. Let $\mathfrak{f}$ be an a-ideal on $X$ and let $f: X \rightarrow Y$ be a function. Then $h(\mathfrak{f})=h(f(\mathfrak{f}))$.

Proof. We see that

$$
h(\mathfrak{f})=\sup _{\phi \in \mathfrak{f}} \inf _{y \in Y} \inf _{x \in f^{-1}\{y\}} \phi(x)=\sup _{\phi \in \mathfrak{f}} \inf _{y \in Y} \phi_{f}(y)=h(f(\mathfrak{f})) .
$$

3. Prime approach ideals. Following Gierz et al. [2] and Lowen et al. [7], we define an a-ideal to be prime if it is a prime ideal in $[0, \infty]^{X}$.

DeFinITION 3.1. An a-ideal $\mathfrak{f}$ on $X$ is said to be prime if for each $\phi, \psi \in[0, \infty]^{X}$ we have

$$
\phi \wedge \psi \in \mathfrak{f} \Longrightarrow \phi \in \mathfrak{f} \text { or } \psi \in \mathfrak{f} .
$$

Prime a-ideals are the numerification of ultrafilters, which is illustrated by the following two propositions.

Proposition 3.2. Let $\mathscr{F}$ be a filter on $X$ and let $\varepsilon<\infty$. Then the following are equivalent:

(1) $\mathscr{F}_{F}$ is an ultrafilter,

(2) $\mathscr{F}_{\varepsilon}$ is prime,

(3) $\mathscr{F}^{\varepsilon}$ is prime.

Proof. To show that $(1) \Rightarrow(2)$, suppose $\phi \wedge \psi \in \mathscr{F}_{\varepsilon}$. Then there is some $F \in \mathscr{F}$ such that $\phi \wedge \psi \leq \varepsilon+\theta_{F}$. Consequently, for every $x \in F$ we have $\phi(x) \leq \varepsilon$ or $\psi(x) \leq \varepsilon$. So, if we put $A:=F \cap\{\phi \leq \varepsilon\}$ and $B:=F \cap\{\psi \leq \varepsilon\}$, then $A \cup B=F$. Since $\mathscr{F}$ is an ultrafilter, $A \in \mathscr{F}$ or $B \in \mathscr{F}$. Because $\phi \leq \varepsilon+\theta_{A}$ and $\psi \leq \varepsilon+\theta_{B}$, this yields the conclusion that $\phi \in \mathscr{F}_{\varepsilon}$ or $\psi \in \mathscr{F}_{\varepsilon}$.

Conversely, to show that (2) $\Rightarrow(1)$, suppose $F \cup G \in \mathscr{F}^{\text {F. Then }}\left(\varepsilon+\theta_{F}\right) \wedge\left(\varepsilon+\theta_{G}\right)=$ $\varepsilon+\theta_{F \cup G} \in \mathscr{F}_{\varepsilon}$, and therefore (because $\mathscr{F}_{\varepsilon}$ is prime) $\varepsilon+\theta_{F} \in \mathscr{F}_{\varepsilon}$ or $\varepsilon+\theta_{G} \in \mathscr{F}_{\varepsilon}$.

Consequently, $F=\left\{\varepsilon+\theta_{F}<\infty\right\} \in\left(\mathscr{F}_{\varepsilon}\right)_{\infty}=\mathscr{F}_{F}$ or $G \in \mathscr{F}$. Thus $\mathscr{F}$ is an ultrafilter. The equivalence of (1) and (3) is shown analogously. 
Proposition 3.3. Let $\mathfrak{f}$ be an a-ideal of bounded height $h$ on $X$. Then $\mathfrak{f}$ is prime if and only if $\mathfrak{f}_{\infty}$ is an ultrafilter.

Proof. To show the only if part, suppose $F \cup G \in \mathfrak{f}_{\infty}$. Then there is some $\phi \in \mathfrak{f}$ such that $\{\phi<\infty\} \subset F \cup G$. If we put $\phi_{1}:=\phi \wedge \theta_{F}$ and $\phi_{2}:=\phi \wedge \theta_{G}$, then $\phi_{1} \wedge \phi_{2}=$ $\phi \wedge \theta_{F \cup G}=\phi$. Since $\mathfrak{f}$ is prime, this means that $\phi_{1} \in \mathfrak{f}$ or $\phi_{2} \in \mathfrak{f}$. Then $F \supset\left\{\phi_{1}<\infty\right\} \in$ $\mathfrak{f}_{\infty}$, and therefore $F \in \mathfrak{f}_{\infty}$ or $G \in \mathfrak{f}_{\infty}$.

In order to show the if part, first notice that for all $\varepsilon \in\left[h, \infty\left[\right.\right.$, we have that $\mathfrak{f}^{\varepsilon} \supset \mathfrak{f}_{\infty}$ and thus, if $\mathfrak{f}_{\infty}$ is an ultrafilter, then $\mathfrak{f}^{h}=\mathfrak{f}_{\infty}$ is an ultrafilter too. From [7, Proposition 1.2.1] we see that $\mathfrak{f}=\left(\mathfrak{f}^{h}\right)_{h}$. Then it follows from Proposition 3.2 that $\mathfrak{f}$ is prime.

Proposition 3.4. Let $\mathfrak{f}$ and $\mathfrak{G}$ be a-ideals on $X$. If $\mathfrak{f}$ is prime and $\mathfrak{f} \subset \mathfrak{G}$, then $\mathfrak{G}$ is prime.

Proof. If $\mathfrak{f}$ is prime, then $\mathfrak{f}_{\infty}$ is an ultrafilter by Proposition 3.3. Clearly, $\mathfrak{f}_{\infty}=\mathfrak{G}_{\infty}$ and, again using Proposition 3.3, this implies that $\mathfrak{6}$ is prime.

We will write

$$
\mathbf{P}(\mathfrak{f}):=\{\mathfrak{P} \mid \mathfrak{P O} \text { is a prime a-ideal and } \mathfrak{f} \subset \mathfrak{P}\} .
$$

The collection $\mathbf{P}(\mathfrak{f})$ is closed under refinement (Proposition 3.4) but it does not have maximal elements: if $x \in X$ and $\alpha<\beta<\infty$, then $\left(\alpha+\theta_{\{x\}}\right)^{\cdot} \subset(\beta+\theta\{x\})^{\cdot}$, both of which are prime. The collection $\mathbf{P}(\mathfrak{f})$ has minimal elements though. These will be investigated later.

Proposition 3.5. Let $\mathfrak{f}$ be an a-ideal on $X$ and let $f: X \rightarrow Y$ be a function. If $\mathfrak{f}$ is prime, then $f(\mathfrak{f})$ is prime too.

Proof. For every $\phi \in \mathfrak{f}$, we have $\left\{\phi_{f}<\infty\right\}=f\{\phi<\infty\}$ and therefore $f(\mathfrak{f})_{\infty}=$ $f\left(\mathfrak{f}_{\infty}\right)$. If $\mathfrak{f}$ is prime, then by Proposition $3.3, \mathfrak{f}_{\infty}$ is an ultrafilter, and thus $f(\mathfrak{f})_{\infty}=$ $f\left(\mathfrak{f}_{\infty}\right)$ is an ultrafilter too. Again by using Proposition 3.3, this means that $f(\mathfrak{f})$ is prime.

As we will see in the sequel, $\mathbf{P}(\mathfrak{f})$ is too big a set for our purposes. Therefore, we will extract a subset of $\mathbf{P}(\mathfrak{f})$ which still contains all the necessary information.

We will consider the set of minimal prime ideals containing $\mathfrak{f}$, that is,

$$
\mathbf{M}(\mathfrak{f}):=\{\mathfrak{P} \in \mathbf{P}(\mathfrak{f}) \mid \mathfrak{P} \text { is minimal }\} .
$$

The fact that $\mathbf{M}(\mathfrak{f})$ is nonempty is a consequence of Zorn's lemma and the following result.

LEMма 3.6. Every totally ordered subcollection of $\mathbf{P}(\mathfrak{f})$ has a lower bound.

Proof. Let $\mathscr{P}$ be a totally ordered subset of $\mathbf{P}(\mathfrak{f})$, and put $\mathfrak{\mathfrak { O } _ { 0 }}:=\cap_{\mathfrak{P} \in \mathscr{P}} \mathfrak{P}$. Obviously, $\mathfrak{P}_{0}$ is an a-ideal and $\mathfrak{f} \subset \mathfrak{P}_{0}$. To see that $\mathfrak{P}_{0}$ is prime, suppose $\phi \wedge \psi \in \mathfrak{H}_{0}$. If for all $\mathfrak{P} \in \mathscr{P}$ we have $\phi \in \mathfrak{P}$, then $\phi \in \mathfrak{P}_{0}$ and we are done. If not, then there exists some $\mathfrak{P} \in \mathscr{P}$ such that $\phi \notin \mathfrak{P}$. Since $\phi \wedge \psi \in \mathfrak{P}$, we must have that $\psi \in \mathscr{Q}$. Then $\psi \in \mathscr{Q}$ for every $\mathscr{Q}_{\mathcal{Q}} \in \mathscr{P}$ such that $\mathscr{Q} \supset \mathfrak{P}$. And if $\mathfrak{Q} \subset \mathfrak{P}$, then $\phi \notin \mathscr{Q}$ and we must again have that $\psi \in \mathfrak{Q}$. Consequently, $\psi \in \mathfrak{P}_{0}$. 
The collection $\mathbf{M}(\mathfrak{f})$ still contains all the relevant information, in the sense of the following proposition.

Proposition 3.7. Let $\mathfrak{f}$ be an a-ideal on $X$. Then

$$
\mathfrak{f}=\bigcap_{\mathfrak{Q} \in \mathbf{M}(\mathfrak{f})} \mathfrak{H}
$$

Proof. We have

$$
\mathfrak{f}=\bigcap_{\mathfrak{P} \in \mathbf{P}(\mathfrak{f})} \mathfrak{P}=\bigcap_{\mathfrak{P} \in \mathbf{M}(\mathfrak{f})} \mathfrak{P} .
$$

The first identity is a well-known fact; the second is a consequence of Lemma 3.6.

In order to show a useful characterization of minimal prime a-ideals (Theorem 3.9), we need one lemma first.

LEMMA 3.8. Let $\mathscr{F}_{\mathfrak{F}}$ be a filter and let $\mathfrak{f}$ be an a-ideal on $X$. If $\mathfrak{f} \vee \mathscr{F}_{0}$ exists, then

$$
\left(\mathfrak{f} \vee \mathscr{F}_{0}\right)_{\infty}=\mathfrak{f}_{\infty} \vee \mathscr{F}
$$

If $\mathscr{F}_{F}$ is an ultrafilter, then $\mathfrak{f} \vee \mathscr{F}_{0}$ exists if and only if $\mathfrak{f}_{\infty} \subset \mathscr{F}$ and then $\left(\mathfrak{f} \vee \mathscr{F}_{0}\right)_{\infty}=\mathscr{F}_{\text {. }}$

Proof. The first part of the lemma follows from the observation that for each $F \in \mathscr{F}$, and for each $\phi \in \mathfrak{f}$, we have $\left\{\phi \vee \theta_{F}<\infty\right\}=\{\phi<\infty\} \cap F$.

In order to prove the second part, suppose $\mathscr{F}$ is an ultrafilter. Then

$$
\begin{aligned}
\mathfrak{f} \vee \mathscr{F}_{0} \text { exists } & \Longleftrightarrow \forall \phi \in \mathfrak{f}, \forall F \in \mathscr{F}_{\mathscr{F}}: \phi \vee \theta_{F} \neq \infty \\
& \Longleftrightarrow \forall \phi \in \mathfrak{f}, \forall F \in \mathscr{F}_{\mathfrak{F}}:\{\phi<\infty\} \cap F \neq \varnothing \\
& \Longleftrightarrow \forall \phi \in \mathfrak{f}:\{\phi<\infty\} \in \mathscr{F} \Longleftrightarrow \mathfrak{f}_{\infty} \subset \mathscr{F} .
\end{aligned}
$$

Then by the first part of the lemma, $\left(\mathfrak{f} \vee \mathscr{F}_{0}\right)_{\infty}=\mathfrak{f}_{\infty} \vee \mathscr{F}=\mathscr{F}$

The following theorem establishes a characterization of minimal a-ideals which will turn out to be of great use in the sequel. If $\mathscr{F}$ is a filter, then we write

$$
\mathbf{U}(\mathscr{F}):=\{\mathcal{U} \mid \mathcal{U} \text { is an ultrafilter and } \mathscr{F} \subset \mathcal{U}\} .
$$

THEOREM 3.9. Let $\mathfrak{f}$ be an a-ideal on $X$. Then

$$
\mathbf{M}(\mathfrak{f})=\left\{\mathfrak{f} \vee \mathcal{U} \mid \boldsymbol{U} \in \mathbf{U}\left(\mathfrak{f}_{\infty}\right)\right\}
$$

Proof. See the proof of Proposition 1.5 in [7].

The next proposition is an illustration of the fact (which we mentioned before) that by considering minimal prime a-ideals instead of prime a-ideals no relevant information gets lost. 
Proposition 3.10. If $\mathfrak{f}$ is an a-ideal on $X$, then there exists some $\mathfrak{f} \in \mathbf{M}(\mathfrak{f})$ such that $h(\mathfrak{f})=h(\mathfrak{P O})$.

Proof. First, suppose that $\mathfrak{f}$ is of bounded height $h$. Let $u$ be an ultrafilter containing $\mathfrak{f}^{h}$ and put $\mathfrak{P}:=\mathfrak{f} \vee \boldsymbol{U}_{0}$. Since $\mathcal{U} \supset \mathfrak{f}^{h} \supset \mathfrak{f}_{\infty}$, we obtain from Theorem 3.9 that $\mathfrak{P} \in \mathbf{M}(\mathfrak{f})$. Obviously, $h(\mathfrak{P}) \geq h$. To show the converse inequality, let $\varepsilon>0$ and $\phi \in \mathfrak{f}$. Since $\{\phi<h+\varepsilon\} \in \mathfrak{f}^{h} \subset \mathcal{U}$, it is possible to find for every $U \in \mathcal{U}$ an element $\sigma_{0}(U) \in U$ such that $\sigma_{0}(U) \in\{\phi<h+\varepsilon\}$. By complete distributivity, we see that

$$
\sup _{U \in \mathscr{U} \mathcal{U}} \inf _{x \in U} \phi(x)=\inf _{\sigma \in \prod_{U \in \mathscr{U} U} U} \sup _{U \in \mathscr{U}} \phi(\sigma(U)) \leq \sup _{U \in \mathscr{U}} \phi\left(\sigma_{0}(U)\right) \leq h+\varepsilon
$$

and therefore (and by arbitrariness of $\varepsilon$ ), we have

$$
h(\not \mathcal{O})=\sup _{\phi \in \mathcal{f}} \sup _{U \in \mathscr{U}} \inf _{x \in X}\left(\phi(x) \vee \theta_{U}(x)\right)=\sup _{\phi \in \mathcal{f}} \sup _{U \in \mathscr{U}} \inf _{x \in U} \phi(x) \leq h .
$$

Second, suppose $\mathfrak{f}$ is of height $\infty$, then let $\mathcal{U}$ be an ultrafilter containing $\mathfrak{f}_{\infty}$ and put $\mathfrak{P}:=\mathfrak{f} \vee U_{0}$. Again, $\mathfrak{f} \in \mathbf{M}(\mathfrak{f})$. Also, we have $h(\mathfrak{P}) \geq h(\mathfrak{f})$, which yields $h(\mathfrak{P})=\infty$.

From Proposition 3.10 we obtain that $h(\mathfrak{f})=\min _{\mathfrak{P} \in \mathbf{M}(\mathfrak{f})} h(\mathfrak{P})$. If we replace the minimum by a supremum, we obtain a new characteristic number for $\mathfrak{f}$.

Definition 3.11. Let $\mathfrak{f}$ be an a-ideal on $X$. Then

$$
m(\mathfrak{f}):=\sup _{\mathfrak{P} \in \mathbf{M}(\mathfrak{f})} h(\mathfrak{P})
$$

is called the prime height of $\mathfrak{f}$.

The following proposition gives a workable description of prime height.

Proposition 3.12. Let $\mathfrak{f}$ be an a-ideal of bounded prime height. Then

$$
m(\mathfrak{f})=\operatorname{supinf}_{\phi \in \mathfrak{f}}\left\{\alpha \mid\{\phi<\alpha\} \in \mathfrak{f}_{\infty}\right\}
$$

Proof. For every $\mu>0$ we have by Theorem 3.9 that

$$
\begin{aligned}
m(\mathfrak{f}) \leq \mu & \Longleftrightarrow \sup _{\mathfrak{Q} \in \mathbf{M}(\mathfrak{f})} h(\mathfrak{P}) \leq \mu \\
& \Longleftrightarrow \sup _{\mathcal{U} \in \mathbf{U}\left(\mathfrak{f}_{\infty}\right)} \sup _{\phi \in \mathfrak{f}} \sup _{U \in \mathscr{U}} \inf _{x \in X}\left(\phi(x) \vee \theta_{U}(x)\right) \leq \mu \\
& \Longleftrightarrow \forall \varepsilon>0, \forall \cup \in \mathbf{U}\left(\mathfrak{f}_{\infty}\right), \forall \phi \in \mathfrak{f}, \forall U \in \mathcal{U}, \exists x \in U: \phi(x)<\mu+\varepsilon \\
& \Longleftrightarrow \forall \varepsilon>0, \forall \cup \in \mathbf{U}\left(\mathfrak{f}_{\infty}\right), \forall \phi \in \mathfrak{f}:\{\phi<\mu+\varepsilon\} \in \mathcal{U} \\
& \Longleftrightarrow \forall \varepsilon>0, \forall \phi \in \mathfrak{f}:\{\phi<\mu+\varepsilon\} \in \mathfrak{f}_{\infty} \\
& \Longleftrightarrow \operatorname{supinf}_{\phi \in \mathfrak{f}}\left\{\alpha \mid\{\phi<\alpha\} \in \mathfrak{f}_{\infty}\right\} \leq \mu
\end{aligned}
$$

which yields the desired result. 
Proposition 3.13. Let $\mathfrak{f}$ be an a-ideal of bounded prime height, let $\mathscr{F}$ be a filter and let $\varepsilon<\infty$. Then we have
(a) $h(\mathfrak{f}) \leq m(\mathfrak{f})$.
(b) If $\mathfrak{f}$ is prime, then $h(\mathfrak{f})=m(\mathfrak{f})$.
(c) $m(\mathfrak{f}) \geq m(\hat{\mathfrak{f}})$.
(d) $m\left(\mathscr{F}_{\varepsilon}\right)=\varepsilon$.
(e) $m\left(\mathscr{F}^{\varepsilon}\right)=\varepsilon$.

Proof. Straightforward verification.

EXAMPLE 3.14. Although equality can occur in Proposition 3.13(c) (as is illustrated in part (d) and (e) of the same proposition), the inequality is strict in general. Suppose $\mathfrak{f}$ is a nontrivial a-ideal on $X$ and $\mathfrak{f}:=\left\{\theta_{F} \wedge \alpha \mid F \in \mathscr{F}, \alpha<\infty\right\}$. Then $\hat{\mathfrak{f}}=\left\{\theta_{F} \mid F \in \mathscr{F}\right\}$. Since $\hat{\mathfrak{f}}=\mathscr{F}_{0}$, we have $m(\hat{\mathfrak{f}})=0$. By Proposition 3.12 however, $m(\hat{\mathfrak{f}})=\infty$.

In order to show an analogue for Proposition 2.15 for prime height, we need to show a couple of lemmas first.

LEMMA 3.15. Let $\mathfrak{f}$ be an a-ideal on $X$ and for each $\mathfrak{P} \in \mathbf{M}(\mathfrak{f})$, let $\phi_{\mathfrak{P}} \in \mathfrak{P}$. Then there exists a finite set $\mathbf{M}_{0} \subset \mathbf{M}(\mathfrak{f})$ such that $\inf _{\mathfrak{P} \in \mathbf{M}_{0}} \phi_{\mathfrak{P}} \in \mathfrak{f}$.

Proof. By Theorem 3.9, it is possible to find $\psi_{\mathfrak{P}} \in \mathfrak{f},{\vartheta_{\mathfrak{P}}}_{\mathfrak{O}} \in \mathbf{U}\left(\mathfrak{f}_{\infty}\right)$ and $U_{\mathfrak{P}} \in \mathcal{U}_{\mathfrak{P}}$ such that $\phi_{\mathfrak{P}} \leq \psi_{\mathfrak{P}} \vee \theta_{U_{\mathfrak{\vartheta}}}$. Then there exists a finite set $\mathbf{M}_{0} \subset \mathbf{M}(\mathfrak{f})$ such that $\cup_{\mathfrak{P} \in \mathbf{M}_{0}} U_{\mathfrak{P}} \in \mathscr{F}_{\infty}$. For suppose it is not, then $\mathfrak{f}_{\infty} \cup\left\{X \backslash U_{\mathfrak{P}} \mid \mathfrak{P} \in \mathbf{M}(\mathfrak{f})\right\}$ has the finite intersection property, and therefore it is contained in some ultrafilter $\mathcal{U} \supset \mathfrak{f}_{\infty}, \mathcal{U}=\mathfrak{U}_{\mathfrak{p}}$ say. But then $X \backslash U_{\mathfrak{p}} \in$ $\boldsymbol{U}_{\mathfrak{B}}$, which is impossible.

Consequently, there exists some $\xi \in \mathfrak{f}$ such that $\{\xi<\infty\} \subset \cup_{\mathfrak{p} \in \mathrm{M}_{0}} U_{\mathfrak{\vartheta}}$. Since

$$
\inf _{\mathfrak{P} \in \mathbf{M}_{0}} \phi_{\mathfrak{P}} \leq \inf _{\mathfrak{P} \in \mathbf{M}_{0}}\left(\psi_{\mathfrak{P}} \vee \theta_{U_{\mathfrak{P}}}\right) \leq \xi \vee\left(\sup _{\mathfrak{P} \in \mathbf{M}_{0}} \psi_{\mathfrak{P}}\right) \in \mathfrak{f},
$$

we have that $\inf _{\mathfrak{P} \in \mathrm{M}_{0}} \phi_{\mathfrak{P}} \in \mathfrak{f}$.

LEMMA 3.16. Let $f$ be an a-ideal on $X$, and let $f: X \rightarrow Y$ be a function. Then

$$
\mathbf{M}(f(\mathfrak{f})) \subset\{f(\mathfrak{P}) \mid \mathfrak{P O} \in \mathbf{M}(\mathfrak{f})\} .
$$

Proof. Suppose $\mathfrak{G} \in \mathbf{M}(f(\mathfrak{f}))$ and for every $\mathfrak{P} \in \mathbf{M}(\mathfrak{f}), f(\mathfrak{P}) \not \subset \mathfrak{G}$. Choose for every $\mathfrak{P} \in \mathbf{M}(\mathfrak{f})$ some $\phi_{\mathfrak{P}}$ such that $\left(\phi_{\mathfrak{P}}\right)_{f} \notin \mathfrak{G}$. By Lemma 3.15 , there is a finite set $\mathbf{M}_{0}$ such that $\inf _{\mathfrak{P} \in \mathbf{M}_{0}} \phi_{\mathfrak{P}} \in \mathfrak{f}$, and thus $\inf _{\mathfrak{P} \in \mathbf{M}_{0}}\left(\phi_{\mathfrak{P}}\right)_{f} \in f(\mathfrak{f}) \subset \mathfrak{G}$. From the fact that $\mathscr{G}$ is prime, it follows that there is some $\mathfrak{P} \in \mathbf{M}(\mathfrak{f})$ such that $\left(\phi_{\mathfrak{P}}\right)_{f} \in \mathfrak{G}$, which is a contradiction. Therefore the existence of some $\mathfrak{P} \in \mathbf{M}(\mathfrak{f})$ such that $f(\mathfrak{P}) \subset \mathfrak{G}$ is guaranteed. Moreover, $f(\mathfrak{Q})$ being prime (by Proposition 3.5) and $\mathfrak{G}$ being minimal prime, we have $f(\mathfrak{Q})=\mathfrak{G}$, which concludes the proof.

Proposition 3.17. Let $\mathfrak{f}$ be an a-ideal on $X$ and let $f: X \rightarrow Y$ be a function. Then $m(f(\mathfrak{f})) \leq m(\mathfrak{f})$.

Proof. Using Lemma 3.16 and Proposition 2.15, respectively, we find that

$$
m(f(\mathfrak{f}))=\sup _{\mathfrak{G} \in \mathbf{M}(f(\mathfrak{f}))} h(\mathfrak{G}) \leq \sup _{\mathfrak{P} \in \mathbf{M}(\mathfrak{f})} h(f(\mathfrak{P}))=\sup _{\mathfrak{A} \in \mathbf{M}(\mathfrak{f})} h(\mathfrak{P})=m(\mathfrak{f}) .
$$


There is no counterpart of Proposition 3.10 for prime height. The following example establishes an a-ideal $\mathfrak{f}$ such that $m(\mathfrak{f})=\infty$, while every $\mathfrak{b} \in \mathbf{M}(\mathfrak{f})$ is of finite height.

EXAMPLE 3.18. For every $n \in \mathbb{N}$, consider

$$
\xi_{n}: \mathbb{R}^{+} \longrightarrow[0, \infty]:\left\{\begin{array}{l}
n \longmapsto n, \\
x \longmapsto 0
\end{array} \text { if } x \neq n\right.
$$

and for every finite $J \subset \mathbb{N}$, let $\xi_{J}:=\sup _{j \in J} \xi_{j}$. Consider the ideal $\mathfrak{f}:=\left\langle\left\{\xi_{J} \mid J \subset\right.\right.$ $\mathbb{N}$ finite $\}$.

First we show that $m(\mathfrak{f})=\infty$. To that end, notice that for every $n \in \mathbb{N}$ we have that $\dot{n} \supset\left\{\mathbb{R}^{+}\right\}=\mathfrak{f}_{\infty}$, and therefore $(\dot{n})_{0} \vee \mathfrak{f} \in \mathbf{M}(\mathfrak{f})$ by Theorem 3.9. A short computation reveals that $h\left((\dot{n})_{0} \vee \mathfrak{f}\right)=n$ and hence $m(\mathfrak{f}) \geq \sup _{n \in \mathbb{N}} h\left((\dot{n})_{0} \vee \mathfrak{f}\right)=\infty$.

Second, we show that for every prime ideal $\mathfrak{P O} \in \mathbf{M}(\mathfrak{f})$, we have $m(\mathfrak{P})<\infty$. Let $\mathcal{U} \in \mathbf{U}\left(\mathfrak{f}_{\infty}\right)$ be such that $\mathfrak{P}=\mathcal{U}_{0} \vee \mathfrak{f}$ and let $\mathscr{H}$ denote the filter generated by the sets $\{k \in \mathbb{N} \mid k \geq n\}$ for all $n \in \mathbb{N}$.

- If $U \supset \mathscr{H}$, then every $U \in U$ is an infinite subset of $\mathbb{N}$, and consequently, $\inf _{x \in U} \xi_{J}(x)=0$ for every $\xi_{J} \in \mathfrak{f}$.

- If $\mathcal{D} \not \mathscr{H}$, then for some $n \in \mathbb{N},\{k \mid k \geq n\} \notin \mathcal{U}$ and thus $U_{0}:=\mathbb{R}^{+} \backslash\{k \mid k \geq n\} \in \mathcal{U}$. Then for every $U \in \mathcal{U}$ such that $U \subset U_{0}$, and for every $\xi_{J} \in \mathfrak{f}$, we see that $\inf _{x \in U} \xi_{J}(x) \leq n$.

In any case, $m(\mathfrak{P})=h(\mathfrak{P})=h\left(\mathcal{U}_{0} \vee \mathfrak{f}\right)<\infty$.

4. Adherence and limit operator. In an approach space $\left(X,(\mathscr{A}(x))_{x \in X}\right)$ for every filter $\mathscr{F}$, Lowen [6] defines its adherence

$$
\alpha \mathscr{F}(x):=\sup _{\eta \in \mathscr{A}(x)} \sup _{F \in \mathscr{F}} \inf _{y \in F} \eta(y)
$$

and its limit

$$
\lambda \mathscr{F}(x):=\sup _{\eta \in \mathscr{A}(x)} \inf _{F \in \mathscr{F}} \sup _{y \in F} \eta(y)=\sup _{u \in \mathbf{U}(\mathscr{F})} \alpha u(x)
$$

In fact, the adherence operator $\alpha$ and the limit operator $\lambda$ both determine the approach structure $(\mathscr{A}(x))_{x \in X}$ (see [6, Propositions 1.8.1 and 1.8.2]). The value $\lambda \mathscr{F}(x)$ (or $\left.\alpha \mathscr{F}(x)\right)$ is interpreted as the distance that the point $x$ is away from being a limit point (cluster point) of $\mathscr{F}$. These notions can be generalized in the setting of a-ideals.

DEFINITION 4.1. Let $\left(X,(\mathscr{A}(x))_{x \in X}\right)$ be an approach space and let $\mathfrak{f}$ be an a-ideal on $X$. Then we define the adherence of $\mathfrak{f}$ by

$$
\alpha \mathfrak{f}(x):=\sup _{\eta \in \mathscr{A}(x)} \sup _{\phi \in \mathfrak{f}} \inf _{y \in X}(\phi+\eta)(y)
$$

and the limit of $f$ by

$$
\lambda \mathfrak{f}(x):=\sup _{\mathfrak{P} \in \mathbf{M}(\mathfrak{f})} \alpha \mathfrak{P}(x), \quad \forall x \in X
$$


We list some basic properties of these operators for future reference and prove some characterizations of the adherence and the limit operator. By abuse of notation, we define $h(\mathfrak{f}+\mathfrak{G})$ to be $\infty$ if $\mathfrak{f}+\mathfrak{G}$ does not exist.

Proposition 4.2. Let $\left(X,(\mathscr{A}(x))_{x \in X}\right)$ be an approach space and let $\mathfrak{f}$ be an a-ideal on $X$. Then $\alpha \mathfrak{f}(x)=h(\mathscr{A}(x)+\mathfrak{f})$ for every $x \in X$.

Proof. It is an immediate consequence of the definitions that for every $x \in X$

$$
\alpha \mathfrak{f}(x)=\sup _{\phi \in \mathfrak{f}} \sup _{\eta \in \mathscr{A}(x)} \inf _{y \in X} \phi+\eta(y)=\sup _{\phi \in \mathfrak{f}+\mathscr{A}(x)} \inf _{y \in X} \phi(y)=h(\mathscr{A}(x)+\mathfrak{f}) .
$$

Proposition 4.3. Let $X$ be an approach space and let $\mathfrak{f}$ and $\mathfrak{6}$ be a-ideals on $X$. Then we have
(a) If $\mathfrak{f} \subset \mathfrak{G}$, then $\alpha \mathfrak{f} \leq \alpha \mathfrak{G}$ and $\lambda \mathfrak{f} \geq \lambda \mathfrak{G}$.
(b) $h(\mathfrak{f}) \leq \alpha \mathfrak{f}$.
(c) $m(\mathfrak{f}) \leq \lambda \mathfrak{f}$.
(d) $\alpha \mathfrak{f} \leq \lambda \mathfrak{f}$.
(e) If $\mathfrak{f}$ is prime, then $\alpha \mathfrak{f}=\lambda \mathfrak{f}$.

Proof. These assertions follow directly from the definitions.

Proposition 4.4. Let $\left(X,(\mathscr{A}(x))_{x \in X}\right)$ be an approach space and let $\mathfrak{f}$ be an a-ideal on $X$. Then

$$
\begin{aligned}
& \lambda \mathfrak{f}(x)=\sup _{\mathfrak{Q} \in \mathbf{M}(\mathfrak{f})} \lambda \mathfrak{P}(x)=\sup _{\mathfrak{P} \in \mathbf{M}(\mathfrak{f})} \alpha \mathfrak{P}(x), \\
& \alpha \mathfrak{f}(x)=\inf _{\mathfrak{P} \in \mathbf{M}(\mathfrak{f})} \lambda \mathfrak{P}(x)=\inf _{\mathfrak{O} \in \mathbf{M}(\mathfrak{f})} \alpha \mathfrak{P}(x) .
\end{aligned}
$$

Proof. The first assertion follows from the definition and Proposition 4.3(e). In order to show the second assertion, notice that from Lemma 3.15 it follows that for any $\sigma \in \prod_{\mathfrak{P} \in \mathbf{M}(\mathfrak{f})} \mathfrak{P}$, there is a finite set $\mathbf{M}_{\sigma} \subset \mathbf{M}(\mathfrak{f})$ such that $\inf _{\mathfrak{P} \in \mathbf{M}_{\sigma}} \sigma(\mathfrak{P} \mathfrak{Q}) \in \mathfrak{f}$. Consequently, we obtain by applying complete distributivity that

$$
\begin{aligned}
& \inf _{\mathfrak{P} \in \mathbf{M}(\mathfrak{f})} \alpha \mathfrak{P}(x)=\inf _{\mathfrak{\vartheta} \in \mathbf{M}(\mathfrak{f})} \sup _{\phi \in \mathfrak{f}} \sup _{\eta \in \mathscr{A}(x)} \inf _{y \in X}(\phi+\eta)(y)
\end{aligned}
$$

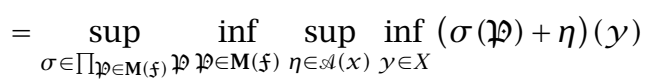

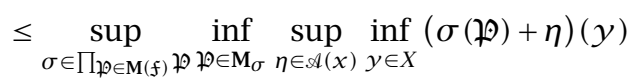

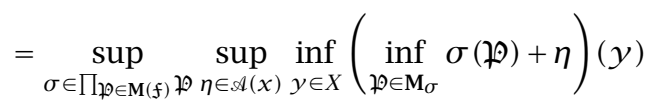

$$
\begin{aligned}
& \leq \alpha \mathfrak{f}(x) \text {. }
\end{aligned}
$$

The other inequality follows from Proposition 4.3(a).

Now it is easy to verify that Definition 4.1 establishes an extension of the adherence and limit of ordinary filters, in the following sense.

Proposition 4.5. Let $\left(X,(\mathscr{A}(x))_{x \in X}\right)$ be an approach space and let $\mathscr{F}$ be a filter on $X$. Then $\alpha \mathscr{F}_{0}=\alpha \mathscr{F}_{\text {and }} \lambda \mathscr{F}_{0}=\lambda \mathscr{F}_{\text {. }}$ 
Proof. Using the characterization of $\mathscr{F}_{0}$ in Proposition 2.11, we see that for every $x \in X$

$$
\begin{aligned}
\alpha \mathscr{F}_{0}(x) & =\sup _{\eta \in \mathscr{A}(x)} \sup _{\phi \in \mathscr{F}_{0}} \inf _{y \in X}(\phi+\eta)(y) \\
& =\sup _{\eta \in \mathscr{A}(x)} \sup _{F \in \mathscr{F}} \inf _{y \in X}\left(\theta_{F}+\eta\right)(y) \\
& =\sup _{\eta \in \mathscr{A}(x)} \sup _{F \in \mathscr{F}} \inf _{y \in F} \eta(y)=\alpha \mathscr{F}(x) .
\end{aligned}
$$

Since $\mathbf{M}\left(\mathscr{F}_{0}\right)=\left\{\mathscr{F}_{0} \vee \mathcal{U}_{0} \mid \mathcal{U} \in \mathbf{U}\left(\left(\mathscr{F}_{0}\right)_{\infty}\right)\right\}=\left\{\boldsymbol{U}_{0} \mid \boldsymbol{U} \in \mathbf{U}(\mathscr{F})\right\}$, we have, by applying the first part of the proposition, that for every $x \in X$

$$
\lambda \mathscr{F}_{0}(x)=\sup _{\mathfrak{Q} \in \mathbf{M}\left(\mathscr{F}_{0}\right)} \alpha \mathfrak{P}(x)=\sup _{u_{U \in \mathbf{U}}(\mathscr{F})} \alpha \bigcup_{0}(x)=\sup _{U \in \mathbf{U}(\mathscr{F})} \alpha \varkappa(x)=\lambda \mathscr{F}(x) .
$$

5. Closure and level-adherence. Yet another characterization of approach spaces can be formulated in terms of the so-called hull operator $\mathrm{h}:[0, \infty]^{X} \rightarrow[0, \infty]^{X}$ defined by

$$
\mathrm{h}(\phi)(x)=\sup _{\eta \in \mathscr{A}(x)} \inf _{y \in X}(\phi+\eta)(y) \quad(\text { for all } x \in X)
$$

(see [6]). The hull operator is a natural quantification of closure in ordinary topology. A function $\phi$ is called regular if $\mathrm{h}(\phi)=\phi$. Regular functions are a generalization of closed sets. Notice that $\alpha \mathfrak{f}=\sup _{\phi \in \mathfrak{f}} \mathrm{h}(\phi)$.

Another numerification of closure and closedness, can be obtained by considering a slight modification of the hull operator, which we will call the closure, defined by

$$
\bar{\phi}(x):=\sup _{\eta \in \mathscr{A}(x)} \inf _{y \in X}(\phi \vee \eta)(y) \quad(\text { for all } x \in X) .
$$

This is essentially not innovating, since we abuse the word "function" in this context as an abbreviation for "sheaf of sets" in the spirit of Proposition 2.9. Nevertheless, the modified concept will prove to be useful in the sequel. If we define the leveladherence of an a-ideal $\mathfrak{f}$ by $\alpha_{l} \mathfrak{f}=\sup _{\phi \in \mathfrak{f}} \bar{\phi}$, then-mutatis mutandis-all the results in the previous section remain true for $\alpha_{l}$ instead of $\alpha$.

The fact that the closure operator too is an extension of closure in topological spaces, which will be a consequence of the following observation.

Proposition 5.1. Let $\left(X,\left(\mathrm{cl}_{\varepsilon}\right)_{\varepsilon}\right)$ be an approach space and let $\phi \in[0, \infty]^{X}$. Then

$$
\{\bar{\phi} \leq \varepsilon\}=\bigcap_{\alpha>\varepsilon} \operatorname{cl}_{\varepsilon}(\{\phi<\alpha\}) \quad \forall \varepsilon \in \mathbb{R}^{+} .
$$

Proof. We have

$$
\begin{aligned}
\bar{\phi}(x) \leq \varepsilon & \Longleftrightarrow \forall \alpha>\varepsilon: \bar{\phi}(x)<\alpha \\
& \Longleftrightarrow \forall \alpha>\varepsilon, \forall \eta \in \mathscr{A}(x), \exists y \in X:(\eta \vee \phi)(y)<\alpha \\
& \Longleftrightarrow \forall \alpha>\varepsilon, \forall \eta \in \mathscr{A}(x):\{\phi<\alpha\} \cap\{\eta<\alpha\} \neq \varnothing \\
& \Longleftrightarrow \forall \alpha>\varepsilon, \forall \eta \in \mathscr{A}(x), \forall \alpha \geq \beta>\varepsilon:\{\phi<\alpha\} \cap\{\eta<\beta\} \neq \varnothing \\
& \Longleftrightarrow \forall \alpha>\varepsilon: x \in \operatorname{cl}_{\varepsilon}(\{\phi<\alpha\}),
\end{aligned}
$$

which yields the result. 
Consequently, we obtain the following result, which holds as well for the hull operator.

COROLLARY 5.2. Let $X$ be a topological approach space. Then $\bar{\theta}_{A}=\theta_{\mathrm{cl}(A)}$ for every $A \subset X$.

Proof. From the definition it is clear that $\bar{\theta}_{A}$ only attains the values 0 and $\infty$. Moreover, by applying Proposition 5.1, we obtain

$$
\bar{\theta}_{A}(x)=0 \Longleftrightarrow x \in \bigcap_{\alpha>0} \operatorname{cl}\left(\left\{\theta_{A}<\alpha\right\}\right) \Longleftrightarrow x \in \operatorname{cl}(A) \Longleftrightarrow \theta_{\mathrm{cl}(A)}(x)=0 .
$$

The closure operator behaves like a topological pre-closure operator (whence the terminology) as is illustrated by the following proposition.

Proposition 5.3. Let $X$ be an approach space let $\phi, \psi,\left(\phi_{i}\right)_{i \in I} \in[0, \infty]^{X}$ and let $A \subset X$. Then

(a) $\bar{\phi} \leq \phi$.

(b) $\overline{\phi \wedge \psi}=\bar{\phi} \wedge \bar{\psi}$.

(c) $\overline{\bigvee_{i \in I} \phi_{i}} \geq \bigvee_{i \in I} \bar{\phi}_{i}$.

(d) $\overline{\varepsilon+\theta_{A}}=\bar{\theta}_{A} \vee \varepsilon$.

Proof. These assertions are easy consequences of the definition.

In general, it is however not true that $\overline{\bar{\phi}}=\bar{\phi}$. Nonetheless, we define the following.

DefinITION 5.4. Let $X$ be an approach space and let $\phi \in[0, \infty]^{X}$. Then $\phi$ is said to be closed if $\bar{\phi}=\phi$.

This is an extension of closedness in topological spaces, in the following sense.

Proposition 5.5. A set $A$ in a topological space is closed if and only if $\theta_{A}$ is closed in the associated topological approach space.

Proof. If $A$ is closed, then by Corollary 5.2 we have that $\bar{\theta}_{A}=\theta_{\operatorname{cl}(A)}=\theta_{A}$, whence $\theta_{A}$ is closed. Conversely, if $\theta_{A}$ is closed, then again by Corollary 5.2, we see that $\theta_{A}=$ $\bar{\theta}_{A}=\theta_{\mathrm{cl}(A)}$, whence $A=\operatorname{cl}(A)$.

From Proposition 5.3, we obtain the following results, which are to be expected.

Proposition 5.6. Let $X$ be an approach space and let $\phi, \psi,\left(\phi_{i}\right)_{i \in I}$ be functions in $[0, \infty]^{X}$. Then

(a) If $\phi$ and $\psi$ are closed, then $\phi \wedge \psi$ is closed.

(b) If for each $i \in I, \phi_{i}$ is closed, then $\bigvee_{i \in I} \phi_{i}$ is closed.

Closed functions turn out to be exactly those functions that have closed sections at every level, whence the terminology.

Proposition 5.7. Let $X$ be an approach space, and let $\phi \in[0, \infty]^{X}$. Then $\phi$ is closed if and only if for every $\varepsilon \in \mathbb{R}^{+},\{\phi \leq \varepsilon\}$ is closed with respect to $\mathrm{cl}_{\varepsilon}$.

Proof. If $\bar{\phi}=\phi$, then for every $\varepsilon \in \mathbb{R}^{+}$we have, by Proposition 5.1, that

$$
\operatorname{cl}_{\varepsilon}(\{\phi \leq \varepsilon\})=\operatorname{cl}_{\varepsilon}\left(\bigcap_{\alpha>\varepsilon}\{\phi<\alpha\}\right) \subset \bigcap_{\alpha>\varepsilon} \operatorname{cl}_{\varepsilon}(\{\phi<\alpha\})=\{\bar{\phi} \leq \varepsilon\}=\{\phi \leq \varepsilon\},
$$


and therefore $\{\phi \leq \varepsilon\}$ is closed with respect to $\mathrm{cl}_{\varepsilon}$. Conversely, let $\varepsilon \in \mathbb{R}^{+}$. By Proposition 5.3(a) we have that $\{\phi \leq \varepsilon\} \subset\{\bar{\phi} \leq \varepsilon\}$. On the other hand, if every $\{\phi \leq \alpha\}$ is closed with respect to $\mathrm{cl}_{\alpha}$, then

$$
\{\bar{\phi} \leq \varepsilon\}=\bigcap_{\alpha>\varepsilon} \operatorname{cl}_{\varepsilon}(\{\phi<\alpha\}) \subset \bigcap_{\alpha>\varepsilon} \operatorname{cl}_{\alpha}(\{\phi \leq \alpha\})=\bigcap_{\alpha>\varepsilon}\{\phi \leq \alpha\}=\{\phi \leq \varepsilon\} .
$$

So $\{\phi \leq \varepsilon\}=\{\bar{\phi} \leq \varepsilon\}$ which, by arbitrariness of $\varepsilon$, means that $\bar{\phi}=\phi$.

Corollary 5.9 shows the relationship between the closure and the hull operator. Given $\phi \in[0, M]^{X}$ for some $M<\infty$, a family $\left(\phi_{\varepsilon}\right)_{\varepsilon>0}$ of functions that attain only finitely many values, is called a development of $\phi$ if for every $\varepsilon>0$ we have that $\phi_{\varepsilon} \leq \phi \leq \phi_{\varepsilon}+\varepsilon$.

LEMMA 5.8. The closure operator is completely determined by the closure of functions of the form $\theta_{A}$. In particular, for any $M<\infty$, any $\phi \in[0, M]^{X}$, and any development $\left(\phi_{\varepsilon}:=\inf _{i=1}^{n(\varepsilon)}\left(m_{i}^{\varepsilon}+\theta_{M_{i}^{\varepsilon}}\right)\right)_{\varepsilon>0}$ of $\phi$, we have that

$$
\bar{\phi}=\sup _{\varepsilon>0} \inf _{i=1}^{n(\varepsilon)}\left(m_{i}^{\varepsilon} \vee \bar{\theta}_{M_{i}^{\varepsilon}}\right) .
$$

Proof. We see, by Proposition 5.3(b) and Proposition 5.3(d), that

$$
\bar{\phi}=\sup _{\varepsilon>0} \bar{\phi}_{\varepsilon}=\sup _{\varepsilon>0} \inf _{i=1}^{n(\varepsilon)}\left(m_{i}^{\varepsilon} \vee \bar{\theta}_{M_{i}^{\varepsilon}}\right) .
$$

COROLlarY 5.9. Let $\phi \in[0, \infty]^{X}$, and for each $N<\infty$ let $\left(\inf _{i=1}^{n(\varepsilon, N)}\left(m_{i}^{\varepsilon, N} \vee \mathrm{h}\left(\theta_{M_{i}^{\varepsilon, N}}\right)\right)\right)_{\varepsilon>0}$ be a development of $\phi \wedge N$. Then

$$
\begin{aligned}
& \bar{\phi}=\sup _{N<\infty} \sup _{\varepsilon>0}\left(\inf _{i=1}^{n(\varepsilon, N)}\left(m_{i}^{\varepsilon, N} \vee \mathrm{h}\left(\theta_{M_{i}^{\varepsilon, N}}\right)\right)\right), \\
& \mathrm{h}(\phi)=\sup _{N<\infty} \sup _{\varepsilon>0}\left(\inf _{i=1}^{n(\varepsilon, N)}\left(m_{i}^{\varepsilon, N}+\bar{\theta}_{M_{i}^{\varepsilon, N}}\right)\right) .
\end{aligned}
$$

Proof. The proof is based on Lemma 5.8, [6, Corollary 1.6.6], and the observation that $\bar{\theta}_{A}=\mathrm{h}\left(\theta_{A}\right)$ for any $A \subset X$.

6. Compactness. The aim of this section is to generalize compactness in topological spaces. Let $X$ be an approach space and let $A \subset X$. Let the collection of all (ordinary) filters on $X$ be denoted by $\mathbf{F}(X)$ and the collection of all filters on $X$ containing $A$ by $\mathbf{F}(A)$. If $\mathscr{F} \in \mathbf{F}(X)$ and for each $F \in \mathscr{F}, F \cap A \neq \varnothing$, then the restriction of $\mathscr{F}$ to $A$ will be denoted by

$$
\mathscr{F} \mid A:=\{F \cap A \mid F \in \mathscr{F}\} .
$$

The measure of compactness of $A$ can be characterized as

$$
\mu_{\mathcal{C}}(A)=\sup _{\mathscr{F} \in \mathbf{F}(X)} \inf _{x \in A} \alpha(\mathscr{F} \mid A)(x)=\sup _{\mathscr{F} \in \mathbf{F}(A)} \inf _{x \in A} \alpha \mathscr{F}(x) .
$$

The number $\mu_{c}(A)$ expresses to what extent the set $A$ differs from being compact. 
If $\mathfrak{f}$ is an a-ideal on $X$ and $\phi \in[0, \infty]^{X}$, and if for all $\psi \in \mathfrak{f}, \phi \vee \psi \neq \infty$, then we define the restriction of $\mathcal{f}$ to $\phi$ by

$$
\mathfrak{f} \mid \phi:=\mathfrak{f} \vee \dot{\phi} .
$$

By abuse of notation, we put $h(\mathfrak{f} \mid \phi):=\infty$ in case $\mathfrak{f} \mid \phi$ does not exist.

A set $A$ is called compact (with respect to a topology) if every filter containing $A$ has an adherence point. We can generalize this notion in the following canonical way.

DEFINITION 6.1. Let $(X, \delta)$ be an approach space and let $\phi \in[0, \infty]^{X}$. Then $\phi$ is said to be compact if and only if for every a-ideal $\mathfrak{f}$ on $X$ we have that $\inf (\phi \vee \alpha \mathfrak{f}) \leq h(\mathfrak{f} \mid \phi)$; and $\phi$ is said to be strongly compact if and only if for every a-ideal $\mathfrak{f}$ on $X$, there is some $x \in X$ such that $\phi(x) \vee \alpha \mathfrak{f}(x) \leq h(\mathfrak{f} \mid \phi)$.

Clearly, if $\phi$ is strongly compact, then $\phi$ is compact. The following proposition pinpoints the precise difference between compactness and strong compactness.

Proposition 6.2. Let $(X, \delta)$ be an approach space and let $\phi \in[0, \infty]^{X}$. Then the following are equivalent:

(1) $\phi$ is strongly compact,

(2) $\phi$ is compact and for every regular function $\psi$, the function $\phi \vee \psi$ attains its minimum.

Proof. To show that $(1) \Rightarrow(2)$, first observe that if $\phi$ is strongly compact, then it must be compact. If $\psi \in[0, \infty]^{X}$, then by (1) there is some $x \in X$ such that $\phi(x) \vee$ $\alpha(\dot{\psi})(x) \leq h(\dot{\psi} \mid \phi)$. So $\psi$ is regular, then

$$
\phi(x) \vee \psi(x)=\phi(x) \vee \mathrm{h}(\psi)(x)=\phi(x) \vee \alpha(\dot{\psi})(x) \leq h(\dot{\psi} \mid \phi)=\inf (\psi \vee \phi) .
$$

To show that (2) $\Rightarrow(1)$, it suffices to remark that $\alpha \mathfrak{f}=\sup _{\xi \in \mathfrak{f}} \mathrm{h}(\xi)$ is a regular function by [6, Definition 1.7.1]. Therefore, and by compactness of $\phi$, there is some $x \in X$ such that $\phi(x) \vee \alpha \mathfrak{f}(x)=\inf (\phi \vee \alpha \mathfrak{f}) \leq h(\mathfrak{f} \mid \phi)$, which shows that $\phi$ is strongly compact.

Therefore, we will not consider strong compactness in the sequel. Most propositions remain true for strong compactness.

On the analogy of equivalent characterizations in the classical case (e.g., $A$ is compact if every ultrafilter containing $A$ converges), here too we can restrict ourselves to particular classes of a-ideals.

THEOREM 6.3. Let $X$ be an approach space and let $\phi \in[0, \infty]^{X}$. The following are equivalent:

(1) $\phi$ is compact.

(2) For every (saturated) prime a-ideal $\mathfrak{f}$ we have inf $(\phi \vee \alpha \mathfrak{f}) \leq h(\mathfrak{f} \mid \phi)$.

(3) For every (saturated) a-ideal $\mathfrak{f}$ containing $\phi$ we have $\inf (\phi \vee \alpha \mathfrak{f}) \leq h(f)$.

(4) For every (saturated) prime a-ideal f containing $\phi$ we have $\inf (\phi \vee \alpha \mathfrak{f}) \leq h(\mathfrak{f})$.

Proof. We will show that $(1) \Rightarrow(2) \Rightarrow(4) \Rightarrow(3) \Rightarrow(1)$, but of course the first two implications are obvious. The question whether or not $\mathfrak{f}$ is saturated is irrelevant, since $h(\hat{\mathfrak{f}})=h(\mathfrak{f})($ Proposition 2.14(b)) and $\alpha \hat{\mathfrak{f}}=\alpha \mathfrak{f}$ (Proposition 4.3(f)). 
To see that (4) $\Rightarrow$ (3), let $\mathfrak{f}$ be an a-ideal containing $\phi$. Choose a prime a-ideal $\mathfrak{P} \in \mathbf{P}(\mathfrak{f})$ such that $h(\mathfrak{f})=h(\mathfrak{P})$, which is possible by Proposition 3.10. Then $\inf (\phi \vee \alpha \mathfrak{f}) \leq$ $\inf (\phi \vee \alpha \mathfrak{Q}) \leq h(\mathfrak{P})=h(\mathfrak{f})$, which we had to prove.

In order to show that (3) $\Rightarrow(1)$, let $\mathfrak{f}$ be an a-ideal. If $h(\mathfrak{f} \mid \phi)=\infty$, then (1) is obvious. So suppose $\mathfrak{f} \mid \phi$ exists and is of bounded height. Then $\phi \in \mathfrak{f} \mid \phi$ and since $\mathfrak{f} \subset \mathfrak{f} \mid \phi$, we find that $\alpha \mathfrak{f} \leq \alpha(\mathfrak{f} \mid \phi)$. Applying (5), we obtain that $\inf (\phi \vee \alpha \mathfrak{f}) \leq \inf (\phi \vee \alpha(\mathfrak{f} \mid$ $\phi)) \leq h(\mathfrak{f} \mid \phi)$, which we had to prove.

In order to generalize the measure of compactness mentioned above, we are to consider the compactness notion associated with the level-adherence operator, which was introduced in the previous section.

DEFINITION 6.4. Let $(X, \delta)$ be an approach space and let $\phi \in[0, \infty]^{X}$. Then $\phi$ is said to be level-compact if and only if for every a-ideal $\mathfrak{f}$ on $X$ we have that $\inf \left(\phi \vee \alpha_{l} \mathfrak{f}\right)$ $\leq h(\mathfrak{f} \mid \phi)$.

The analogue of Theorem 6.3 remains true for level-compactness. Moreover, we have the following relationship between Definition 6.1 and the measure of compactness.

Proposition 6.5. Let $X$ be an approach space, and let $A \subset X$. Then for every $\varepsilon>0$, $\mu_{c}(A) \leq \varepsilon$ if and only if $\varepsilon+\theta_{A}$ is level-compact. Consequently, $\mu_{c}(A)=\inf \{\varepsilon \mid \varepsilon+$ $\theta_{A}$ is level-compact $\}$.

Proof. Since $\varepsilon \leq h\left(\mathfrak{f} \mid \varepsilon+\theta_{A}\right)$ for every a-ideal $\mathfrak{f}$, compactness of $\varepsilon+\theta_{A}$ comes down to the fact that for every $\mathfrak{f}$ we have $\inf _{x \in A} \alpha \mathfrak{f}(x) \leq h\left(\mathfrak{f} \mid \varepsilon+\theta_{A}\right)$, that is,

$$
\inf _{x \in A} \sup _{\phi \in \mathfrak{f}} \sup _{\eta \in \mathscr{A}(x)} \inf _{y \in X}(\eta \vee \phi)(y) \leq \sup _{\phi \in \mathfrak{f}} \inf _{y \in X} \phi(y) \vee \varepsilon
$$

Now, to see the if part, let $\mathscr{F} \in \mathbf{F}(X)$ and consider $\mathfrak{f}:=(\mathscr{F} \mid A)_{0}$. Then $\inf _{x \in A} \sup _{F \in \mathscr{F}} \sup _{\eta \in \mathscr{A}(x)} \inf _{y \in A \cap F} \eta(y) \leq \varepsilon$, or $\inf _{x \in A} \alpha\left(\mathscr{F}_{F} \mid A\right)(x) \leq \varepsilon$. Hence, $\mu_{c}(A)=$ $\sup _{\mathscr{F} \in \mathbf{F}(X)} \inf _{x \in X} \alpha(\mathscr{F} \mid A)(x) \leq \varepsilon$.

Conversely, to show the only if part, let $\mathfrak{f}$ be an a-ideal on $X$. Consider $\mathscr{F}_{\mathscr{F}}:=\mathfrak{f}_{\varepsilon}$. Then $\inf _{x \in A} \sup _{\eta \in \mathscr{A}(x)} \sup _{\phi \in \mathcal{f}} \inf _{y \in A \cap\{\phi \leq \varepsilon\}} \eta(y) \leq \varepsilon$ and therefore

$$
\begin{aligned}
\inf _{x \in A} \sup _{\eta \in \mathscr{A}(x)} \sup _{\phi \in \mathcal{f}} \inf _{y \in X}(\eta \vee \phi)(y) & \leq \inf _{x \in A} \sup _{\eta \in \mathscr{A}(x)} \sup _{\phi \in \mathfrak{f}} \inf _{y \in A \cap \phi \leq \varepsilon}(\eta \vee \phi)(y) \\
& \leq \sup _{\phi \in \mathfrak{f}} \inf _{y \in A} \phi(y) \vee \varepsilon,
\end{aligned}
$$

which we had to show.

We can generalize different well-known results on compact sets. For instance, the intersection of a finite number of compact sets is again compact.

Proposition 6.6. Let $X$ be an approach space and let $\phi, \psi \in[0, \infty]^{X}$. If $\phi$ and $\psi$ are (level-)compact, then $\phi \wedge \psi$ is (level-)compact. 
Proof. Let $\mathfrak{f}$ be a prime a-ideal containing $\phi \wedge \psi$. Then $\phi \in \mathfrak{f}$ or $\psi \in \mathfrak{f}$. Suppose $\phi \in \mathfrak{f}$. Then since $\phi$ is compact, we have that $\inf ((\phi \wedge \psi) \vee \alpha \mathfrak{f}) \leq \inf (\phi \vee \alpha \mathfrak{f}) \leq h(\mathfrak{f})$. By Theorem 6.3, this means that $\phi \wedge \psi$ is compact.

An analogous argument proves the statement for level-compactness.

COROLLARY 6.7. Let $X$ be an approach space and let $A, B \subset X$. Then $\mu_{c}(A \cup B) \leq$ $\mu_{c}(A) \vee \mu_{c}(B)$. Consequently, in a topological space, the union of two compact sets is compact, and, in a metric space, the union of two totally bounded sets is totally bounded.

Proof. If $\mu_{c}(A) \vee \mu_{c}(B) \leq \varepsilon$, then $\mu_{c}(A) \leq \varepsilon$ and $\mu_{c}(B) \leq \varepsilon$ and then $\varepsilon+\theta_{A}$ and $\varepsilon+\theta_{B}$ are level-compact by Proposition 6.5. Calling on Proposition 6.6 we see that $\varepsilon+\theta_{A \cup B}=\left(\varepsilon+\theta_{A}\right) \wedge\left(\varepsilon+\theta_{B}\right)$ is level-compact, and thus $\mu_{c}(A \cup B) \leq \varepsilon$. Therefore, $\mu_{c}(A \cup B) \leq \mu_{c}(A) \vee \mu_{c}(B)$.

Suppose $A$ and $B$ are compact in some topological space. Then we obtain from [4] that $\mu_{c}(A)=\mu_{c}(B)=0$ in the associated topological approach space, and thus $\mu_{c}(A \cup B)=0$ by the first part of the proposition. This yields the compactness of $A \cup B$.

An analogous argument holds for total boundedness in metric spaces.

Proposition 6.8. Let $X$ be an approach space and let $\phi, \psi \in[0, \infty]^{X}$. If $\phi$ is regular (closed) and $\psi$ is (level-)compact, then $\phi \vee \psi$ is (level-)compact.

Proof. For every $\xi \in[0, \infty]^{X}$, we have that $\mathrm{h}(\phi \vee \xi) \geq \mathrm{h}(\phi) \vee \mathrm{h}(\xi)=\phi \vee \mathrm{h}(\xi)$. Therefore, for every a-ideal $\mathfrak{f}$ on $X$, we have

$$
\begin{aligned}
\inf ((\phi \vee \psi) \vee \alpha \mathfrak{f}) & =\inf \left(\phi \vee \psi \vee \sup _{\xi \in \mathfrak{f}} h(\xi)\right)=\inf \sup _{\xi \in \mathfrak{f}}(\phi \vee \psi \vee \mathrm{h}(\xi)) \\
& \leq \inf \sup _{\xi \in \mathfrak{f}}(\psi \vee \mathrm{h}(\phi \vee \xi))=\inf (\psi \vee \alpha(\mathfrak{f} \mid \phi)) \\
& \leq h((\mathfrak{f} \mid \phi) \mid \psi)=h(\mathfrak{f} \mid(\phi \vee \psi))
\end{aligned}
$$

whence $\phi \vee \psi$ is compact.

COROLLARY 6.9. Let $X$ be an approach space and let $A, B \subset X$. If $B$ is closed with respect to the topological reflection of $X$, then $\mu_{c}(A \cap B) \leq \mu_{c}(A)$. Consequently, in $a$ topological space, the intersection of a compact set and a closed set is compact.

Proof. If $\mu_{c}(A) \leq \varepsilon$, then $\varepsilon+\theta_{A}$ is level-compact (Proposition 6.5) and by Proposition $5.7 \theta_{B}$ is closed. Consequently, $\varepsilon+\theta_{A \cap B}=\theta_{B} \vee\left(\varepsilon+\theta_{A}\right)$ is level-compact.

Proposition 6.10. Let $X$ and $Y$ be approach spaces, let $f: X \rightarrow Y$ be a contraction and let $\phi \in[0, \infty]^{X}$. If $\phi$ is (level-)compact, then $\phi_{f}$ is (level-)compact.

Proof. Let $\mathfrak{G}$ be an a-ideal on $Y$. If $h\left(\mathfrak{G} \mid \phi_{f}\right)=\infty$, then there is nothing to prove. So suppose $h\left(\mathfrak{G} \mid \phi_{f}\right)<\infty$. Then $f^{-1}(\mathfrak{G})$ exists, since

$$
\begin{aligned}
\operatorname{supinf}_{\psi \in \mathfrak{G}}(\phi \vee \psi \circ f) & =\sup _{\psi \in \mathfrak{G}} \inf _{x \in X}\left(\phi_{f}(f(x)) \vee \psi(f(x))\right) \\
& =\sup _{\psi \in \mathfrak{G}} \inf _{y \in Y}\left(\phi_{f}(y) \vee \psi(y)\right)=h\left(\mathfrak{G} \mid \phi_{f}\right),
\end{aligned}
$$


and moreover, $h\left(f^{-1}(\mathfrak{G}) \mid \phi\right)=h\left(\mathfrak{G} \mid \phi_{f}\right)$. Then

$$
\begin{aligned}
\inf \left(\phi_{f} \vee \alpha \mathscr{G}\right) & =\inf \left(\phi_{f} \vee \operatorname{suph}_{\psi \in \mathfrak{G}} h^{\prime}(\psi)\right)=\inf _{x \in X}\left(\phi_{f}(f(x)) \vee \operatorname{suph}_{\psi \in \mathfrak{G}}(\psi)(f(x))\right) \\
& \leq \inf _{x \in X}\left(\phi_{f}(f(x)) \vee \sup _{\psi \in \mathfrak{G}}(\psi \circ f)(x)\right)=\inf _{x \in X}\left(\phi(x) \vee \alpha\left(f^{-1}(\mathfrak{G})\right)(x)\right) \\
& =h\left(f^{-1}(\mathfrak{G}) \mid \phi\right)=h\left(\mathfrak{G} \mid \phi_{f}\right) .
\end{aligned}
$$

Hence, $\phi_{f}$ is compact.

COROLLARY 6.11. Let $X$ and $Y$ be approach spaces, let $f: X \rightarrow Y$ be a contraction, and let $A \subset X$. Then $\mu_{c}(f(A)) \leq \mu_{c}(A)$. Consequently, in a topological space, the continuous image of a compact set is compact.

Proof. The proof goes along the same lines as the proof of Corollary 6.9.

Finally, we want to show a Tychonoff-like theorem. To that end, we need two lemmas. If for each $i \in I, \phi_{i} \in[0, \infty]^{X_{i}}$, then define $\prod_{i \in I} \phi_{i} \in[0, \infty] \Pi X_{i}$ by $\prod_{i \in I} \phi_{i}(x)=\sup _{i \in I} \phi_{i}\left(x_{i}\right)$. The canonical projections will be denoted by $\pi_{j}: \prod_{i \in I} X_{i} \rightarrow$ $X_{j}: x=\left(x_{i}\right)_{i} \mapsto x_{j}$. If $\mathfrak{f}$ is an a-ideal on $\prod_{i \in I} X_{i}$, then we write $\mathfrak{f}_{i}:=\pi_{i}(\mathfrak{f})$ for every $i \in I$. If different approach spaces $X_{1}, X_{2}, \ldots$ are involved, we will denote all their adherence operators by $\alpha$, all the hull operators by $\mathrm{h}$ and so on, in order to avoid involved notation.

LEMMA 6.12. For every $i \in I$, let $X_{i}$ be an approach space, and let $\mathfrak{f}$ be a prime a-ideal on $\prod_{i \in I} X_{i}$. Then $\alpha \mathfrak{f}=\prod_{i \in I} \alpha \mathfrak{f}_{i}$.

Proof. Suppose $\phi \in \mathfrak{f}$. If for every $i \in I, \mu_{i}$ is a regular function on $X$, then for every finite $J \subset I$ we have that $\mu:=\inf _{i \in J} \mu_{i} \circ \pi_{i}$ is a regular function on $\prod_{i \in I} X_{i}$. Let $\mathscr{R}$ denote the collection of all regular functions $\mu \leq \phi$ that can be constructed in this manner. If $\mu \in \mathscr{R}$, then $\mu \in \mathfrak{f}$ and by primality of $\mathfrak{f}$, there is some $j \in J$ such that $\mu_{j} \circ \pi_{j} \in \mathfrak{f}$, and so $\mu_{j} \in \mathfrak{f}_{j}$. Consequently, $\mu \leq \mu_{j} \circ \pi_{j}=\mathrm{h}\left(\mu_{j}\right) \circ \pi_{j} \leq \alpha \mathfrak{f}_{j} \circ \pi_{j} \leq \prod_{i \in I} \alpha \mathfrak{f}_{i}$. Hence, $\alpha \mathfrak{f}=\sup _{\mu \in \mathscr{R}} \mu \leq \prod_{i \in I} \alpha \mathfrak{f}_{i}$.

Conversely, to show the other inequality, let $\phi \in \mathfrak{f}$. Observe that for every $i \in I$ and every $\phi_{i}:=\phi_{\pi_{i}} \in[0, \infty]^{X_{i}}$ the function $\mathrm{h}\left(\phi_{i}\right) \circ \pi_{i}$ is regular. Since $\mathrm{h}\left(\phi_{i}\right) \circ \pi_{i} \leq$ $\phi_{i} \circ \pi_{i} \leq \phi$, we obtain that $\mathrm{h}\left(\phi_{i}\right) \circ \pi_{i} \leq \mathrm{h}(\phi)$, and thus $\alpha \mathfrak{f}_{i} \circ \pi_{i}=\left(\sup _{\phi \in \mathfrak{f}} \mathrm{h}\left(\phi_{i}\right)\right) \circ \pi_{i}=$ $\sup _{\phi \in \mathfrak{f}} \mathrm{h}\left(\phi_{i}\right) \circ \pi_{i} \leq \sup _{\phi \in \mathfrak{f}}=\alpha \mathfrak{f}$, whence $\prod_{i \in I} \alpha \mathfrak{f}_{i}=\sup _{i \in I} \alpha \mathfrak{f}_{i} \circ \pi_{i} \leq \alpha \mathfrak{f}$.

LEMMA 6.13. For every $i \in I$, let $\phi_{i} \in[0, \infty]^{X_{i}}$ and let $\mathfrak{f}$ be an a-ideal on $\prod_{i \in I} X_{i}$. Then $h\left(\mathfrak{f} \mid \prod_{i \in I} \phi_{i}\right) \geq \sup _{i \in I} h\left(\mathfrak{f}_{i} \mid \phi_{i}\right)$.

Proof. Let $\phi=\prod_{i \in I} \phi_{i}, X=\prod_{i \in I} X_{i}$ and let $\mathfrak{f}_{i}:=\pi_{i}(\mathfrak{f})$. Suppose $\boldsymbol{\xi} \in \mathfrak{f}$. Since $\phi_{i} \leq \phi_{\pi_{i}}$, we find that $\phi_{i} \vee \xi_{\pi_{i}} \leq \phi_{\pi_{i}} \vee \xi_{\pi_{i}} \leq(\phi \vee \xi)_{\pi_{i}}$. Therefore, $h\left(\mathfrak{f}_{j} \mid \phi_{j}\right)=$ $\sup _{\xi \in f} \inf _{y \in X_{i}} \phi_{i} \vee \xi_{\pi_{i}}(y) \leq \sup _{\xi \in f} \inf _{y \in X_{i}}(\phi \vee \xi)_{\pi_{i}}(y)=\sup _{\xi \in f} \inf _{x \in X}(\phi \vee \xi)(x)=$ $h(\mathfrak{f} \mid \phi)$. By arbitrariness of $i \in I$, this proves the claim.

THEOREM 6.14. For every $i \in I$, let $X_{i}$ be an approach space and $\phi_{i} \in[0, \infty]^{X_{i}}$. If $\phi_{i}$ is compact for all $i \in I$, then $\prod_{i \in I} \phi_{i}$ is compact. Conversely, if $\prod_{i \in I} \phi_{i}$ is compact and $\inf \phi_{i}=0$ for all $i \in I$, then $\phi_{i}$ is compact for all $i \in I$. 
Proof. Write $\phi:=\prod_{i \in I} \phi_{i}$ and $X=\prod_{i \in I} X_{i}$. Let $\mathfrak{f}$ be a prime ideal on $X$ and let $\varepsilon>0$. If for every $i \in I, \phi_{i}$ is compact, then $\inf \left(\phi_{i} \vee \alpha \mathfrak{f}_{i}\right) \leq h\left(\mathfrak{f}_{i} \mid \phi_{i}\right)$ by definition. Choose some $x=\left(x_{i}\right)_{i} \in X$ such that for every $i \in I, \phi_{i}\left(x_{i}\right) \vee \alpha \mathfrak{f}_{i}\left(x_{i}\right)<h\left(\mathfrak{f}_{i} \mid \phi_{i}\right)+\varepsilon$. Then, calling on Lemmas 6.12 and 6.13 , respectively, we see that

$$
\begin{aligned}
\inf (\phi \vee \alpha \mathfrak{f}) & \leq \phi(x) \vee \alpha \mathfrak{f}(x)=\sup _{i \in I} \phi_{i}\left(x_{i}\right) \vee \sup _{i \in I} \alpha \mathfrak{f}_{i}\left(x_{i}\right) \\
& \leq \sup _{i \in I} h\left(\mathfrak{f}_{i} \mid \phi_{i}\right)+\varepsilon \leq h(\mathfrak{f} \mid \phi)+\varepsilon,
\end{aligned}
$$

which by arbitrariness of $\varepsilon$ proves that $\phi$ is compact.

To show the second part of the assertion, we will show that under the condition that $\inf \phi_{j}=0$ we have that $\phi_{j}=\phi_{\pi_{j}}$. It is easy to see that $\phi_{j} \leq \phi_{\pi_{j}}$. Conversely, let $\varepsilon>0$ and let $y_{j} \in X_{j}$ be arbitrary. For every $i \in I \backslash\{j\}$, choose some $y_{i} \in X_{i}$ such that $\phi_{i}\left(y_{i}\right) \leq \varepsilon$ and write $y=\left(y_{i}\right)_{i}$. Then $\phi_{\pi_{j}}\left(y_{j}\right) \leq \phi(y)=\sup _{i \in I} \phi_{i}\left(y_{i}\right)=$ $\sup _{i \in I \backslash\{j\}} \phi_{i}\left(y_{i}\right) \vee \phi_{j}\left(y_{j}\right) \leq \varepsilon \vee \phi_{j}\left(y_{j}\right)$. By arbitrariness of $\varepsilon$, this yields $\phi_{\pi_{j}}\left(y_{j}\right) \leq \phi_{j}$.

So, if $\phi$ is compact then every $\phi_{j}=\phi_{\pi_{j}}$ is compact by Proposition 6.10.

For the converse implication, the extra condition that all $\phi_{i}$ "have zero height" is necessary. For instance, if $\phi=\infty$ and $\psi$ is not compact, then $\phi \times \psi=\infty$ is nonetheless compact.

COROLLARY 6.15. A product of topological spaces is compact if and only if each factor space is compact.

QUESTION 6.16. Is there a Tychonoff-like theorem for level-compactness?

\section{REFERENCES}

[1] J. Banaś and K. Goebel, Measures of Noncompactness in Banach Spaces, Lecture Notes in Pure and Applied Mathematics, vol. 60, Marcel Dekker, New York, 1980. MR 82f:47066. Zbl 441.47056.

[2] G. Gierz, K. H. Hofmann, K. Keimel, J. D. Lawson, M. W. Mislove, and D. S. Scott, A Compendium of Continuous Lattices, Springer-Verlag, Berlin, 1980. MR 82h:06005. Zbl 452.06001.

[3] K. Kuratowski, Sur les espaces complets, Fund. Math. 15 (1930), 301-309 (French).

[4] R. Lowen, Kuratowski's measure of noncompactness revisited, Quart. J. Math. Oxford Ser. (2) 39 (1988), no. 154, 235-254. MR 89i:54010. Zbl 672.54025.

[5] _ Approach spaces: a common supercategory of TOP and MET, Math. Nachr. 141 (1989), 183-226. MR 90i:54025. Zbl 676.54012.

[6] _ Approach Spaces: the Missing Link in the Topology-Uniformity-Metric Triad, Oxford Mathematical Monographs, Oxford University Press, New York, 1997. MR 98f:54002. Zbl 891.54001.

[7] R. Lowen, M. Sioen, and C. Verbeeck, Generalizing filter convergence by ideals, submitted.

[8] R. Lowen and B. Windels, AUnif: a common supercategory of pMET and Unif, Int. J. Math. Math. Sci. 21 (1998), no. 1, 1-18. MR 98j:54014. Zbl 890.54024.

ROBERT LOWEN: DePARTMENT OF MATHEMATICS AND COMPUTER SCIENCE, UNIVERSITY OF ANTWERP-RUCA, GROENENBORGERLAAN 171, 2020 ANTWERPEN, BELGIUM

E-mail address: 1owen@ruca. ua. ac . be

BART Windels: DePARTMENT OF MATHEMATICS AND COMPUTER SCIENCE, UNIVERSITY OF ANTWERP-RUCA, GROENENBORGERLAAN 171, 2020 ANTWERPEN, BELGIUM

E-mail address: winde1s@ruca.ua.ac.be

URL: http://math. ruca.ua.ac . be/TOP 


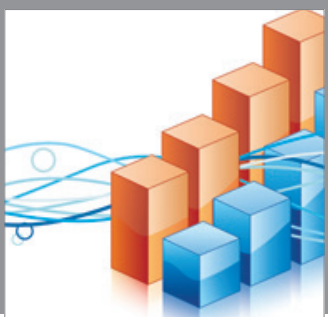

Advances in

Operations Research

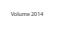

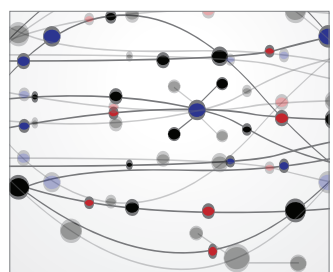

\section{The Scientific} World Journal
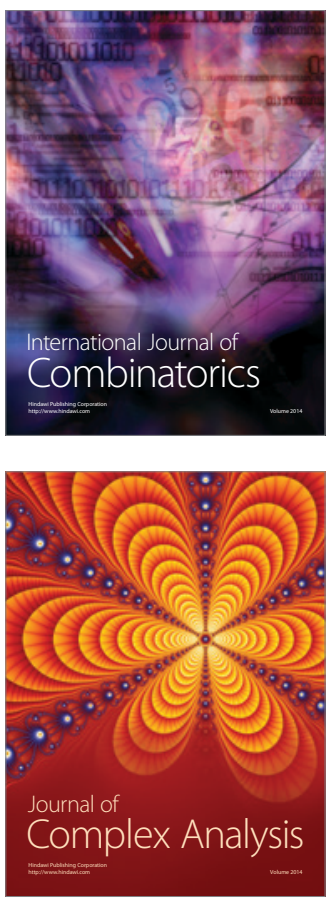

International Journal of

Mathematics and

Mathematical

Sciences
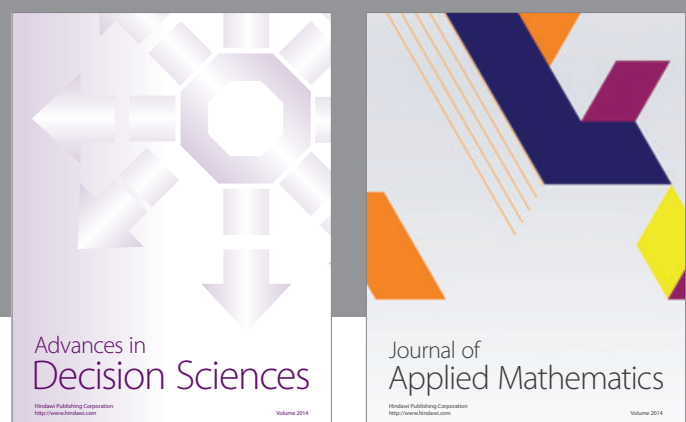

Journal of

Applied Mathematics
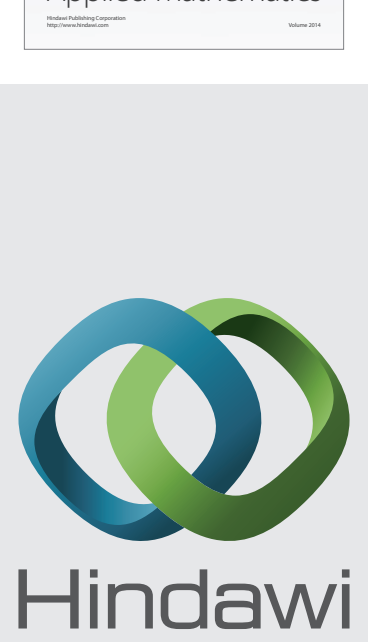

Submit your manuscripts at http://www.hindawi.com
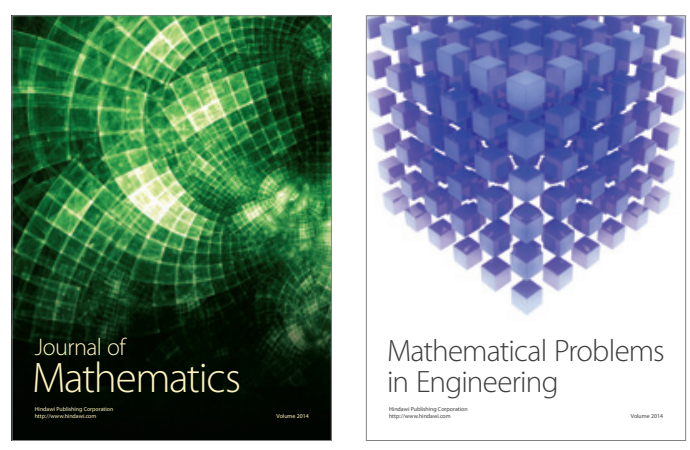

Mathematical Problems in Engineering
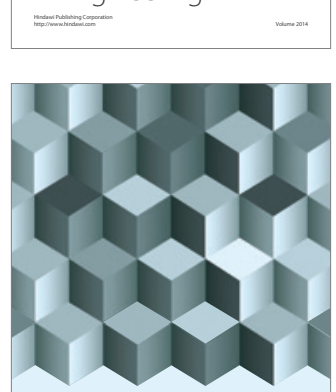

Journal of

Function Spaces
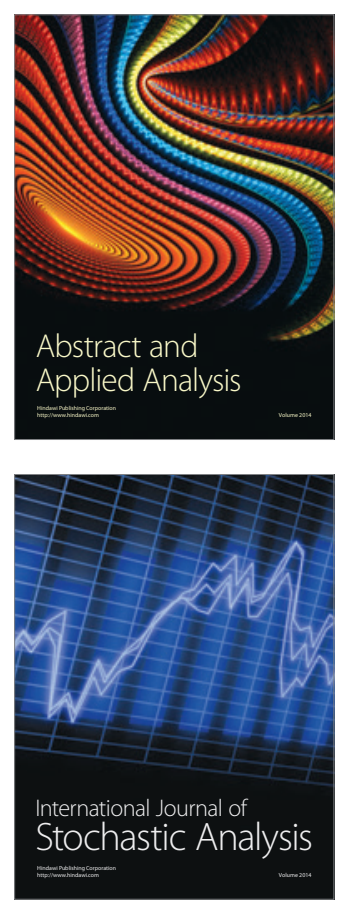

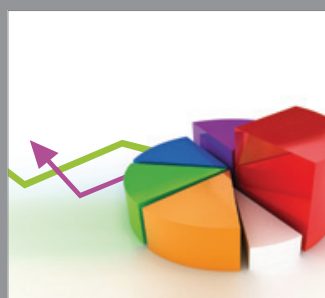

ournal of

Probability and Statistics

Promensencen
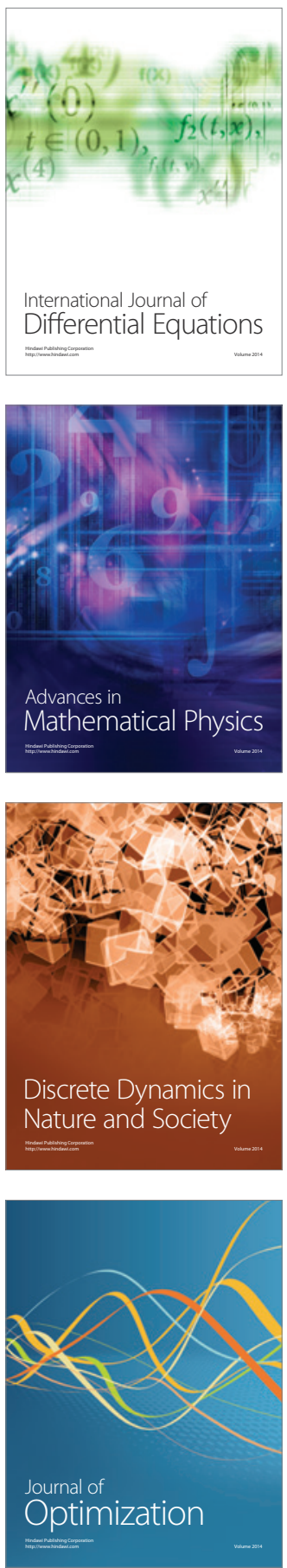